\title{
Migration(en) im Geschichte- und Politikunterricht
}

\author{
Eine Analyse der Darstellung von Migration(en) in Schulbüchern für \\ Geschichte und Sozialkunde/Politische Bildung der Sekundarstufe I
}

\section{Christian Filko ${ }^{1}$}

https://doi.org/10.53349/resource.2021.i16.a993

\section{Zusammenfassung}

Eine vom damaligen Bundesministerium für Unterricht, Kunst und Kultur in Auftrag gegebene und zwischen 2011 und 2013 durchgeführte Studie zur Darstellung von Migration(en) in Geographie- und Geschichte-Schulbüchern zeigte auf, dass Migration in Schulbüchern größtenteils problematisiert und als ein Phänomen der Anderen dargestellt wird, welches in Österreich zu Herausforderungen im Hinblick auf Integration führt (Hintermann et al., 2013). Diese Ergebnisse, die die Binnenmigration und Österreich als Schauplatz von Migration nicht thematisieren sowie positive Effekte von Migrationsbewegungen ignorieren, bilden den Ausgangspunkt für den vorliegenden Beitrag. Mit der Einführung des kompetenzorientierten, modularisierten Lehrplans für das Unterrichtsfach Geschichte und Sozialkunde/Politische Bildung (GSP2) im Jahr 2016 (BMBF, 2016) wurde mit dem Modul Migration die Querschnittsthematik erstmalig im GSP-Lehrplan der Sekundarstufe I verankert. Da die Schulbücher lehrplankonform verfasst werden müssen, kam es zu zahlreichen Neuauflagen bereits vorhandener Serien, zur Einstellung einiger Schulbuchserien sowie zur Entstehung neuer Schulbuchreihen im Zuge der Lehrplanreform. Der vorliegende Beitrag argumentiert, dass durch die Verankerung des Themenbereichs Migration im Lehrplan in den neu approbierten Schulbüchern ein im Vergleich zu den Studienergebnissen von Hintermann et al. (2013) differenzierteres Bild von Migration(en) gezeichnet wird. Hierzu wird nach einem knappen Einblick in den Forschungsstand eine Lehrplananalyse durchgeführt, ehe die Ergebnisse der mittels qualitativer Inhaltsanalyse durchgeführten Schulbuchanalyse präsentiert werden.

Schlüsselwörter:

Migration

Schulbuchanalyse

Geschichtsdidaktik

Politikdidaktik

\author{
Keywords: \\ Migration \\ Textbook analysis \\ Didactics of history \\ Didactics of civic education
}

\section{Einleitung}

Migration prägt wie kaum ein anderes Phänomen die Menschheitsgeschichte, welche stets von Wanderungen gekennzeichnet war, sodass sich bei näherer Betrachtung die Menschheitsgeschichte als Migrationsgeschichte beschreiben lässt. Das „menschliche Muster“ der Migration (Liebig, 2007, S. 7) wird zunehmend zum Thema politischer Auseinandersetzungen - neben der Politisierung lässt sich jedoch auch eine gesellschaftliche Polarisierung in Bezug auf die Migrationsthematik feststellen. Daher ist insbesondere die Institution Schule in ihrem politischen Bildungsauftrag gefragt, den Kindern und Jugendlichen ein wissenschaftsbasiertes Verständnis von Migration(en) zu vermitteln und im Sinne des zentralen geschichts- und politikdidaktischen Prinzips der Multiperspektivität (vgl. Gautschi, 2009; Pandel, 2012; Sander, 2014) verschiedene Standpunkte zum Thema näher zu bringen, um die Lernenden schließlich zu einer eigenständigen Urteilsbildung anzuleiten. Wenngleich die Politische Bildung als Unterrichtsprinzip verankert ist (vgl. BMBF, 2015), entfällt gerade in der Sekundarstufe ein Großteil der politischen Bildungsarbeit auf das Kombinationsfach Geschichte und Sozialkunde/Politische

\footnotetext{
1 Pädagogische Hochschule Wien, Grenzackerstraße 18, 1100 Wien.

E-Mail: christian.filko@phwien.ac.at

${ }^{2}$ Im Folgenden wird das Unterrichtsfach Geschichte und Sozialkunde/Politische Bildung aus Gründen der einfacheren Lesbarkeit mit GSP abgekürzt.
} 
Bildung (vgl. Hellmuth \& Klepp, 2010, S. 132ff.) und inhaltlich benachbarte Fächer, wie beispielsweise Geographie und Wirtschaftskunde sowie Deutsch, Religion und Psychologie und Philosophie, die als Trägerfächer der Politischen Bildung fungieren (vgl. Hämmerle et al., 2009; Sander, 2012).

Im GSP-Unterricht kommt den Schulbüchern bei der Wissens- und Kompetenzvermittlung eine besondere Bedeutung zu, da sie wie kaum ein anderes Medium den Unterricht beeinflussen und sowohl bei Schüler*innen als auch Lehrer*innen hohes Vertrauen genießen (vgl. SORA, 2014; Kipman \& Kühberger, 2018). Als „heimlicher Lehrplan“ bilden Schulbücher - und nicht die curricularen Vorgaben des Lehrplans - eines der primären Instrumente zur Unterrichtsvorbereitung der Lehrpersonen (vgl. SORA, 2014). Aus diesen Gründen, die die Macht der in Schulbüchern vermittelten Bilder und Inhalte untermauern, ergibt sich der Analysebedarf ebendieser. In den folgenden Abschnitten des Artikels werden zunächst die bisherigen Forschungsergebnisse zum Thema Migration(en) in Schulbüchern präsentiert, da sie den Ausgangspunkt für die vorliegende Untersuchung bilden. Es folgt eine knappe Curriculumanalyse des GSP-Lehrplans der Sekundarstufe I, welche den Materialkorpus einschränkt. Nach einer Erläuterung der der Schulbuchanalyse zugrundeliegenden Methode der qualitativen Inhaltsanalyse erfolgt die Darstellung und Diskussion der zentralen Ergebnisse der durchgeführten Studie.

\section{Migration(en) im Schulbuch: Undifferenziert und problematisiert?}

Eine von Christiane Hintermann, Christa Markom und Heidemarie Weinhäupl im Zeitraum von 2011 bis 2013 durchgeführte Studie zu Migration(en) im Schulbuch zeigt eine unzureichende und problematisierende Behandlung von Migration in Geographie- und Geschichte-Schulbüchern der Sekundarstufe auf (vgl. Hintermann et al., 2013). Die Auseinandersetzung mit der Thematik erfolgt in den analysierten Werken nicht breit genug: Es wird beispielsweise nicht auf die Bedeutung von Binnenmigration - die zahlenmäßig häufigste Migrationsform eingegangen und die Geschichte Österreichs wird als keine Migrationsgeschichte dargestellt. Vielmehr fungiert Migration als eine „additional story“ und wird als eine zusätzliche Erzählung, die unabhängig von der Geschichte Österreichs ist, präsentiert. Eine weitere festgestellte Tendenz stellt die Problematisierung von Migration in den analysierten Lehrwerken dar. Anstatt Migration als Bereicherung zu sehen oder die Potenziale und Probleme, die durch Migration entstehen können, ausgewogen darzustellen, zeigt sich ein Problemdiskurs vorherrschend:

In nahezu allen untersuchten Schulbüchern herrschte bezüglich des Themas Migration ein Problemdiskurs vor [...]. So wurde in einigen Büchern Migration als Bedrohung oder Gefahr dargestellt. [...] Auch im Kontext der österreichischen Migrationsgeschichte fanden sich Sprachbilder, die die ein Bedrohungsszenario erzeugen [...]. (Markom \& Weinhäupl, 2013, S. 16)

Dementsprechend wird durch viele der analysierten Schulbücher - entgegen dem zentralen geschichtsdidaktischen Prinzip der Multiperspektivität - eine einseitige Darstellung von Migration vorgenommen. Neben der fehlenden Multiperspektivität lässt sich auch die Rhetorik, mit der „ein Bedrohungsszenario" erzeugt wird, kritisieren, da diese populistisch und unwissenschaftlich ist. Ähnliche Ergebnisse weisen in der Bundesrepublik Deutschland durchgeführte Schulbuchanalysen zum Thema Migration auf. So stellt Helmut Geuenich in seiner Untersuchung nordrhein-westfälischer Geschichte- und Politikschulbücher der Sekundarstufe I fest, dass Migration problematisiert wird und Migrant*innen als homogene Gruppe dargestellt werden (vgl. Geuenich, 2015, S. 355). Philipp Ströhle schlussfolgert in einer Schulbuchstudie, deren Analysekorpus niedersächsische Schulbücher der Fächer Erdkunde, Gemeinschaftskunde und Geschichte der Sekundarstufe II umfasst: „Die untersuchten Schulbücher zeichnen fast ausnahmslos ein im Spannungsverhältnis zwischen Problem- und Bereicherungsdiskurs eingebettetes Bild der Migration, wobei die Problematisierungen deutlich dominieren" (Ströhle, 2014, S. 133). Sowohl die österreichische Studie von Hintermann et al. (2013) als auch die deutschen Studien von Geuenich (2015) und Ströhle (2014) kritisieren zudem den rund um das Thema Migration geführten Nützlichkeitsdiskurs, durch welchen Migrant*innen im Sinne einer ökonomischen Verwertungslogik in nützliche und weniger nützliche Gruppen eingeteilt werden.

Die Ergebnisse, die die Studien zur Behandlung des Themenbereiches Migration in Lehrwerken aufzeigten, könnten ein Grund dafür gewesen sein, dass Migration als eigenständiges Modul im neuen GSP-Lehrplan (BMBF, 2016) verankert wurde, wie der folgende Abschnitt zeigt. 


\section{Curriculare Vorgaben im Lehrplan 2016: Migration als Querschnittsthema}

Mit dem kompetenzorientierten und modularisierten Lehrplan 2016 kam es erstmalig zur Verankerung von „Migration“ als eigenständigem Modul im Lehrplan des Unterrichtsfaches Geschichte und Sozialkunde/Politische Bildung in der Sekundarstufe I (vgl. BMBF, 2016). Zuvor wurde Migration nur implizit und marginal in den GSPSchulbüchern der Sekundarstufe I behandelt, wie die Vorgängerstudie von Hintermann et al. (2013) zeigt. Der neue Lehrplan 2016 bricht zudem die bis dato vorherrschende chronologische Bearbeitung historischer Themen auf und ermöglicht neben Querschnitten auch Längsschnitte, welche den globalen Aspekt von Migration thematisieren. Konkret findet sich die Thematik Migration schwerpunktmäßig in Modul 5 der siebenten Schulstufe, welches der historischen Bildung zugeordnet wird, wieder. Der zeitliche Rahmen wird den Angaben des Lehrplans folgend vom 19. Jahrhundert bis zur Gegenwart gespannt und schließt damit auch aktuelle Migrationsbewegungen mit ein. In der Kompetenzkonkretisierung und der thematischen Konkretisierung werden die Modulziele aus inhaltlich-thematischer wie methodisch-didaktischer Sicht näher ausformuliert:

\begin{tabular}{|c|c|}
\hline Kompetenzkonkretisierung & Thematische Konkretisierung \\
\hline $\begin{array}{l}\text { - } \text { „Schriftliche und bildliche Quellen } \\
\text { beschreiben, analysieren und } \\
\text { interpretieren } \\
\text { - } \quad \text { Perspektivität von Quellen } \\
\text { wahrnehmen } \\
\text { - } \quad \text { Eigene historische Erzählungen } \\
\text { erstellen } \\
\text { - Erkenntnisse aus der Arbeit mit } \\
\text { Quellen und Darstellungen für die } \\
\text { eigene Orientierung nutzen“ } \\
\text { (BMBF, 2016, o.S.) }\end{array}$ & $\begin{array}{l}\text { - } \quad \text { Die Begriffe Migration, Asyl und Integration } \\
\text { erarbeiten } \\
\text { - Weltweite Migrationsbewegungen } \\
\text { vergleichen und Ursachen ermitteln } \\
\text { - Durch Migration entstehende } \\
\text { Herausforderungen in Auswanderungs- und } \\
\text { Einwanderungsländern analysieren und mögliche } \\
\text { Lösungen diskutieren } \\
\text { Migration am Beispiel von Lebensgeschichten } \\
\text { vom 19. Jahrhundert bis in die Gegenwart } \\
\text { darstellen“ (BMBF, 2016, o.S.) }\end{array}$ \\
\hline
\end{tabular}

Tabelle 1: Kompetenz- und thematische Konkretisierung des Moduls 5 der siebenten Schulstufe (BMBF, 2016, o.S.)

Migration soll folglich anhand schriftlicher und bildlicher Quellen bearbeitet werden, wobei auch die Perspektivität der Quellen thematisiert werden soll. Eine präzise Begriffsdefinition relevanter Termini wie Migration selbst, aber auch Asyl und Integration soll erfolgen. Der globale Aspekt der Beschäftigung mit Migration wird stark hervorgehoben, indem „weltweite Migrationsbewegungen“ verglichen werden sollen. Neben diesem Querschnitt wird auch ein zeitlich begrenzter Längsschnitt gefordert, da Migration „vom 19. Jahrhundert bis in die Gegenwart" untersucht werden soll. Der Lehrplan geht auch auf die Multiperspektivität ein, indem einerseits als Kompetenzkonkretisierung das Wahrnehmen der „Perspektivität von Quellen“ gefordert wird und andererseits „Migration am Beispiel von Lebensgeschichten“ behandelt werden soll. Diese Einbeziehung biographischer Elemente führt dazu, dass nicht nur über Migrant*innen gesprochen wird, sondern auch diese selbst durch Quellen zur Sprache kommen. Schließlich gilt es, die „Erkenntnisse aus der Arbeit mit Quellen und Darstellungen“ im Sinne der politischen Urteilskompetenz „für die eigene Orientierung [zu] nutzen“ und migrationsbedingte Herausforderungen lösungsorientiert zu diskutieren (BMBF 2016, o.S.). Inwiefern die im Lehrplan gelegte breite Palette an Wissensbereichen und Kompetenzen in den Schulbüchern umgesetzt wird, zeigen die durchgeführten Schulbuchanalysen.

\section{Schulbuchanalyse: Methodischer Zugang und Material}

Die sozialwissenschaftliche und didaktische Schulbuchforschung erfreut sich zunehmender Beliebtheit, wobei sich drei verschiedene Zugänge unterscheiden lassen: die prozessorientierte, die produktorientierte und die wirkungsorientierte Schulbuchforschung. Erstere fokussiert auf den Entstehungsprozess der Lehrwerke, während Letztere die Wirkung von Schulbüchern bzw. deren Inhalten auf Schüler*innen untersucht. Die vorliegende Schulbuchanalyse lässt sich hingegen eindeutig der produktorientierten Schulbuchanalyse zuordnen, da die Inhalte des Lehrwerkes im Mittelpunkt der Analyse stehen (vgl. Weinbrenner, 1995, S. 36). Für einen solchen auf den Inhalt fokussierten Zugang liegt als Erhebungs- und Analyseinstrument die qualitative Inhaltsanalyse nach Mayring nahe (vgl. Handro \& Schönemann, 2011), da sich diese als „empirische Methode zur systematischen, intersubjektiv nachvollziehbaren Beschreibung inhaltlicher und formaler Merkmale von 
Mitteilungen“ (Früh, 2017, 33) besonders eignet, größere Mengen von Daten zu bearbeiten (Mayring, $2015 b$, S. 474). Die Auswahl des Analysekorpus beruht auf einer aus dem BMBWF schriftlich eingeholten Information über die meist bestellten Schulbuchserien in der Sekundarstufe I. Lehrwerke, die nicht dem Lehrplan 2016 entsprechen, wurden aufgrund der fehlenden systematischen Thematisierung von Migration aus der Analyse ausgeschlossen, während erst im Zuge des Lehrplans 2016 erschienene Schulbuchserien in die Analyse miteinbezogen wurden, auch wenn sie nicht unter den meistbestellten Schulbüchern gelistet waren. Somit konnte sichergestellt werden, dass die Schulbuchanalyse sowohl stark verbreitete Lehrwerke, die durch ihre hohen Bestellzahlen einen hohen Wirkungsradius haben, da sie eine Vielzahl an Schüler*innen erreichen, als auch im Zuge der Lehrplanänderung neu entstandene Lehrwerke miteinschließt und dadurch aussagekräftig ist.

In einem Mixed-Method-Design wurden neben qualitativen vereinzelt auch quantitative Inhaltsanalysen durchgeführt, wobei letztere Analysen rein deskriptiver Natur sind. Der Fokus der Studie lag auf qualitativen Inhaltsanalysen, die dem Ablaufmodell der allgemeinen Inhaltsanalyse nach Mayring folgend durchgeführt wurden (vgl. Mayring, 2015a, S. 62). Dabei erfolgte die Festlegung der Analyseeinheiten deduktiv, ergo durch ein vorab festgelegtes Kategoriensystem. Im Zuge der Codierung wurden die Schulbuchtexte mehrfach gelesen und auf Basis der im Codierleitfaden festgelegten Ankerbeispiele den entsprechenden Kategorien zugeordnet (vgl. Mayring, 2015a, S. 51). Eine abschließende induktive Codierschleife stellte sicher, dass auch die in den Schulbüchern vorhandenen, aber im Codierleitfaden nicht berücksichtigten Inhalte Eingang in die Analyse fanden. Zum methodischen Vorgehen bleibt anzumerken, dass die qualitative Inhaltsanalyse trotz mehrfacher Überarbeitungsschleifen einen subjektiven Zugang darstellt, der jedoch durch die Offenlegung der Vorgehensweise und die Erstellung eines Codierleitfadens als wissenschaftlich nachvollziehbares Vorgehen gilt (vgl. Mayring, 2015b, S. 474).

\section{Darstellung und Diskussion der Ergebnisse}

In diesem Abschnitt werden die zentralen Ergebnisse der durchgeführten Schulbuchanalysen dargestellt und diskutiert. Um eine möglichst breite Präsentation der Ergebnisse zu ermöglichen, werden die Ergebnisse der qualitativen und quantitativ-deskriptiven Analysen nicht getrennt voneinander, sondern thematisch geclustert dargestellt. Die sieben analysierten Lehrwerke werden aus Gründen der Anonymisierung mit dem Kürzel $L$ für Lehrwerk und den Ziffern 1 bis 7 bezeichnet. Im Folgenden werden die Ergebnisse hinsichtlich (1) der Definition von Migration und Flucht, (2) der Nennung unterschiedlicher Formen von Migration, (3) der Darstellung unterschiedlicher Ursachen von Migration, (4) der Behandlung wesentlicher Migrationsereignisse und (5) der Thematisierung von Migration als historischem und politischem Thema dargelegt und diskutiert.

\subsection{Definition von Migration und Flucht}

Im Gegensatz zu der von Hintermann et al. (2013) festgestellten Unschärfe in der Verwendung der Termini Migration, Migrant*in, Flucht und Asyl wird in allen analysierten Lehrwerken eine Definition des Begriffes Migration vorgenommen. Dabei erfolgt in allen Schulbüchern die Herleitung des Begriffes aus dem Lateinischen und eine Übersetzung mit ,Wanderung'. Einige Lehrwerke bieten den Schüler*innen mehrere Definitionen von Migration - darunter auch jene der Vereinten Nationen - an und fordern sie auf, selbstständig eine Definition zu formulieren. Nur in einem Schulbuch wird die Übersetzung von Migration als Wanderung kritisch betrachtet:

Migration oder Wanderung? Bis ins 20. Jahrhundert nannte man Migrationsbewegungen ,Wanderungen' oder ,Völkerwanderungen'. Diese Begriffe haben jedoch wenig mit der Wirklichkeit zu tun. [...] Bei Wanderungen kehrt man wieder an den Ausgangsort zurück. Migration meinte eine endgültige oder zumindest langfristige Verlagerung des Wohnortes oder des Lebensmittelpunktes. (L2, S. 80)

In nahezu allen Schulbüchern wird zudem implizit oder explizit zwischen Migration und Asyl differenziert. Drei Lehrwerke bieten eine explizite Differenzierung, welche beispielsweise durch Gegenüberstellung erfolgt, wie folgendes Beispiel aus L6 zeigt: 


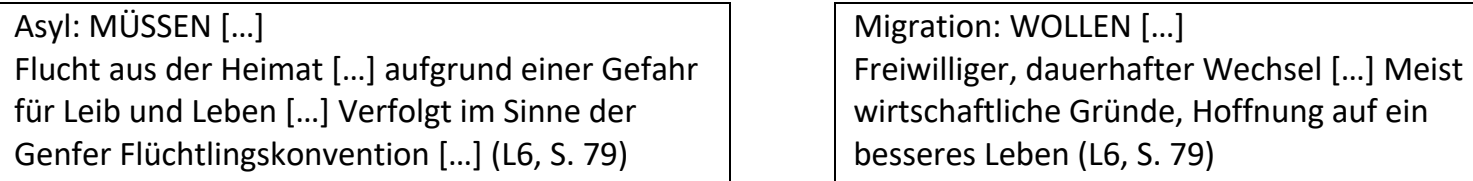

Migration: WOLLEN [...]

Freiwilliger, dauerhafter Wechsel [...] Meist wirtschaftliche Gründe, Hoffnung auf ein

besseres Leben (L6, S. 79)

Tabelle 2: Gegenüberstellung von Migration und Flucht im Lehrwerk L6 (vereinfacht nach L6, S. 79)

In drei weiteren Lehrwerken erfolgt eine implizite Differenzierung zwischen Migration und Flucht. Hierbei werden keine direkten Vergleiche vorgenommen, jedoch ist anhand unterschiedlicher Unterkapitel oder aufgrund der konkreten Definition von Migration oder Flucht eine Differenzierung vorhanden.

\subsection{Formen der Migration}

Neben der Differenzierung zwischen Migration und Flucht gilt es auch die verschiedenen Formen der Migration aufzuzeigen, um ein breites Verständnis des Phänomens zu ermöglichen. Hierbei ist unter anderem zwischen Binnenmigration und internationaler Migration einerseits und zwischen Emigration und Immigration andererseits zu unterscheiden.

Der Begriff der Binnenmigration wird in vier der analysierten Lehrwerke (L2, $L 4, L 6, L 7)$ definiert, jedoch führt nur eines davon (L2) den Begriff der internationalen Migration als Pendant zur Binnenmigration an. In einem der Lehrwerke wird zwar der Gegensatz zwischen den beiden Migrationsformen hergestellt und auch auf die höhere Häufigkeit von Binnenmigration hingewiesen, jedoch erfolgt dennoch keine Definition der internationalen Migration: „Binnenmigration [...] Verlegt jemand seinen Wohnsitz innerhalb ein und desselben Staates, so spricht man von Binnenmigration. Diese ist wesentlich häufiger als internationale Migration“ (L4, S. 87). Zudem ist die fehlende Perspektive von Binnenmigration innerhalb der Europäischen Union zu kritisieren. Nur L2 führt die UNHCR-Definition von Migration an, die ein Verständnis von Migration innerhalb der EU als Binnenmigration fördert. Die Lehrwerke L1, L3 und L5 thematisieren weder die Binnen- noch die internationale Migration.

Einen weiteren wesentlichen Aspekt, der maßgeblich für das Verständnis von Migration ist, bildet die Unterteilung von Migration in den Prozess der Emigration und der Immigration. Auch diese wird lediglich in vier der untersuchten Schulbücher (L2, L4, L5, L6) vorgenommen, wobei Emigration mit Ab- oder Auswanderung und Immigration mit Ein- oder Zuwanderung übersetzt wird. In $\mathrm{L} 5$ wird neben der Definition der beiden Begriffe auch ihr Zusammenhang verdeutlicht, indem die Wechselseitigkeit der Emigrations- und Immigrationsprozesse beleuchtet wird und die beiden Migrationsformen so als zwei Seiten einer Medaille dargestellt werden (vgl. L2, S. 129). Die Lehrwerke L1, L3 und L7 thematisieren Emigration und Immigration weder explizit noch implizit.

\subsection{Ursachen der Migration}

Da die Gründe für Migration vielfältig sind, ist diese Breite auch in den Schulbüchern entsprechend zu repräsentieren. In allen untersuchten Lehrwerken findet sich eine Darstellung vielfältiger Migrationsursachen. In allen Lehrwerken außer $\mathrm{L} 3$ wird einleitend darauf aufmerksam gemacht, dass es unterschiedliche und vielfältige Gründe gibt, die Menschen dazu veranlassen, zu migrieren, wie die folgenden Ausschnitte zeigen:

„Menschen verlassen oft ihre Heimat. Kriege, Hunger, Umweltkatastrophen oder Arbeitslosigkeit sind die häufigsten Gründe dafür." (L1, S. 88)

"Menschen haben verschiedene Gründe, warum sie ihre Heimat verlassen." (L2, S. 82)

"Migration wird auch immer von verschiedenen Faktoren bestimmt [...]." (L4, S. 87)

"Manche Menschen treffen selbst die Entscheidung, woanders hinzuziehen (z.B. wegen Arbeit, Ausbildung oder Familie). Andere werden durch unzumutbare Lebensbedingungen (z.B. Krieg, Hungersnot) oder Verfolgung dazu gezwungen, ihre Heimat zu verlassen.“ (L5, S. 127)

„Es gibt vielfältige Ursachen für die Migrationsströme vom 19. Jh. bis zur Gegenwart.“ (L6, S. 76)

„Es gibt viele Gründe, warum sich Menschen dazu entscheiden, ihre Heimat zu verlassen.“ $(\mathrm{L7}, 140)$ 
Die Autor*innen von L3 wählen hingegen eine implizite Vorgehensweise: So wird nicht eingangs auf die Vielzahl von Migrationsursachen hingewiesen, stattdessen werden im Laufe des Kapitels jeweils mit historischen Ereignissen verknüpft verschiedene Migrationsursachen angeführt. Vier Lehrwerke (L2, L4, L5, L7) nutzen zudem auch die Push- und Pullfaktoren, um Migrationsmotive zu erklären. Die folgende Tabelle gibt einen Überblick in Hinsicht auf die Nennung verschiedener Ursachen der Migration in den einzelnen Lehrwerken. Ein X im jeweiligen Feld verweist auf die explizite Behandlung des Migrationsgrundes. Felder, die $(X)$ enthalten, beschreiben eine implizite Thematisierung der Migrationsursache, beispielsweise durch Nennung in einem Fallbeispiel, ohne näher auf die Migrationsursache einzugehen. Leere Felder bedeuten eine fehlende Thematisierung.

\begin{tabular}{|c|c|c|c|c|c|c|c|}
\hline Politische Gründe & L1 & L2 & L3 & L4 & L5 & L6 & L7 \\
\hline politische Verfolgung & $x$ & $x$ & & $x$ & $x$ & & $(X)$ \\
\hline instabiles politisches System & & & & $x$ & & & \\
\hline Kriege, Konflikte & $x$ & $x$ & $x$ & $x$ & $x$ & $x$ & $x$ \\
\hline religiöse Verfolgung & $x$ & $x$ & $x$ & $x$ & $x$ & & $(X)$ \\
\hline Zugehörigkeit zu Minderheit & $x$ & & $x$ & & $x$ & $(\mathrm{X})$ & $(X)$ \\
\hline politische Stabilität (Zielland) & & $x$ & & $x$ & & $x$ & \\
\hline Wirtschaftliche Gründe & L1 & L2 & L3 & L4 & L5 & L6 & L7 \\
\hline Armut, Hunger, Obdachlosigkeit & $x$ & $x$ & $x$ & $x$ & $x$ & & $x$ \\
\hline Arbeitslosigkeit & $x$ & & $x$ & $x$ & $x$ & $x$ & $x$ \\
\hline Arbeitsplätze (Zielland) & $x$ & $x$ & $x$ & $x$ & $x$ & $x$ & $x$ \\
\hline fehlende (Aus-)Bildungschancen & $x$ & & & & $x$ & $x$ & $x$ \\
\hline gutes Sozialsystem (Zielland) & $\mathrm{x}$ & & & $(\mathrm{X})$ & $\mathrm{x}$ & $\mathrm{x}$ & \\
\hline Ökologische Gründe & L1 & L2 & L3 & L4 & L5 & L6 & L7 \\
\hline Natur- und Umweltkatastrophen & $x$ & & & $x$ & $x$ & $x$ & $x$ \\
\hline Gesellschaftliche Gründe & L1 & L2 & L3 & L4 & L5 & L6 & L7 \\
\hline Überbevölkerung & & & $x$ & & & & \\
\hline Bevölkerungsrückgang (Zielland) & & & & & & $x$ & \\
\hline Persönliche Gründe & L1 & $\mathrm{L} 2$ & L3 & L4 & L5 & L6 & L7 \\
\hline Familienzusammenführung & & & $x$ & & & $(\mathrm{X})$ & $x$ \\
\hline Abenteuerlust & $x$ & & & & & $x$ & $x$ \\
\hline Hoffnung auf ein besseres Leben & & & $\mathrm{x}$ & $(\mathrm{X})$ & $x$ & & $\mathrm{x}$ \\
\hline
\end{tabular}


Tabelle 3: Nennung der Migrationsursachen in den Lehrwerken (eigene Darstellung)

Aus der Tabelle ist zu entnehmen, dass in den Schulbüchern vielfältige Ursachen der Migration aufgezeigt werden und mit Ausnahme von L2 (6 Nennungen) alle Lehrwerke 9 oder mehr Migrationsursachen anführen. Politische und wirtschaftliche Motive für Migration werden im Gegensatz zu ökologischen, gesellschaftlichen und persönlichen Gründen in allen Schulbüchern angeführt. Interessant erscheint, dass Ursachen, die ein tiefergehendes Verständnis von Migration ermöglichen, wie beispielsweise die Instabilität des politischen Systems im Herkunftsland oder demographische Entwicklungen im Herkunfts- (Bevölkerungszunahme) und Zielland (Bevölkerungsrückgang), in nur wenigen Lehrwerken behandelt werden.

\subsection{Zentrale Migrationsereignisse}

Der Lehrplan definiert als Modulziel die Behandlung der „Migrationsgeschichte vom 19. Jahrhundert bis zur Gegenwart“ (BMBF, 2016, o.S.). Dementsprechend wurden die Schulbücher in Hinsicht auf zuvor definierte und aus der Literatur gewonnenen Schlüsselereignisse der Migrationsgeschichte des 19., 20. und 21. Jahrhunderts mit Bezug zu Österreich analysiert. Die folgende Tabelle zeigt die Ergebnisse dieser Analyse. Ein X im Feld zeigt die Thematisierung des Migrationsereignisses im jeweiligen Lehrwerk auf. Felder mit $(X)$ bedeuten, dass das Migrationsereignis zwar beispielsweise auf einem Zeitstrahl oder durch ein Bild erwähnt, aber nicht näher behandelt wird. Leere Felder verweisen auf eine fehlende Behandlung des Migrationsereignisses.

\begin{tabular}{|c|c|c|c|c|c|c|c|}
\hline Vor dem 19. Jahrhundert & L1 & L2 & L3 & L4 & L5 & L6 & L7 \\
\hline $\begin{array}{l}\text { Migrationsbewegungen vor dem } 19 . \\
\text { Jahrhundert (lehrplanmäßig nicht } \\
\text { vorgesehen) }\end{array}$ & $\mathrm{x}$ & $\mathrm{x}$ & $\mathrm{x}$ & & $\mathrm{x}$ & $x$ & \\
\hline 19. Jahrhundert & L1 & L2 & L3 & L4 & L5 & L6 & L7 \\
\hline $\begin{array}{l}\text { Binnenmigration in der } \\
\text { Habsburgermonarchie }\end{array}$ & & $\mathrm{x}$ & $\mathrm{x}$ & $\mathrm{x}$ & $\mathrm{x}$ & $\mathrm{x}$ & \\
\hline Transatlantische Emigration & $x$ & $x$ & $x$ & $x$ & $x$ & $x$ & $x$ \\
\hline 20. Jahrhundert & L1 & L2 & L3 & L4 & L5 & L6 & L7 \\
\hline Transatlantische Emigration & $x$ & $x$ & $x$ & $x$ & $x$ & $x$ & $x$ \\
\hline Antisemitische Flucht/Vertreibung & $x$ & & $x$ & & $x$ & & \\
\hline \multicolumn{8}{|l|}{ Displaced Persons } \\
\hline Deutschsprachige Minderheiten & $x$ & $x$ & $x$ & & & & \\
\hline Ungarnaufstand 1956 & $(X)$ & & $x$ & & & & $(X)$ \\
\hline Prager Frühling 1968 & & & $x$ & & & & $(X)$ \\
\hline Gastarbeiter 1960er/1970er-Jahre & $x$ & & $\mathrm{x}$ & $\mathrm{x}$ & & $\mathrm{x}$ & $x$ \\
\hline Jugoslawienkriege & $x$ & & $\mathrm{x}$ & & $\mathrm{x}$ & & $(\mathrm{X})$ \\
\hline
\end{tabular}


R\&E-SOURCE $h$ ttps://journal.ph-noe.ac.at Online Journal for Research and Education Ausgabe 16, Oktober 2021, ISSN: 2313-1640

\begin{tabular}{|l|c|c|c|c|c|c|c|}
\hline Kosovokrieg 1999 & & & & & & & \\
\hline 21. Jahrhundert & L1 & L2 & L3 & L4 & L5 & L6 & L7 \\
\hline Zuwanderung aus dem Osten & & & (X) & & & & \\
\hline EU-Binnenwanderung & & & & & & & \\
\hline Zuwanderung aus Afrika/Asien & $\mathrm{X}$ & & $\mathrm{X})$ & & & & \\
\hline Fluchtbewegungen aus Syrien & $\mathrm{X}$ & $\mathrm{X}$ & $\mathrm{X}$ & $\mathrm{X}$ & $\mathrm{X}$ & $\mathrm{X}$ & $\mathrm{X}$ \\
\hline
\end{tabular}

Tabelle 4: Nennung der Migrationsereignisse in den Lehrwerken (eigene Darstellung)

Entgegen den Bestimmungen des Lehrplans (vgl. BMBF, 2016) werden in fünf der sieben Schulbücher auch Migrationsereignisse, die zeitlich vor dem 19. Jahrhundert liegen, behandelt, was jedoch aus geschichtsdidaktischer Sicht und im Sinne des konzeptuellen Lernens (vgl. Germ, 2015) sinnvoll erscheint, da an das historische Vorwissen der Schüler*innen angeknüpft wird. In allen Lehrwerken wird die transatlantische Migration aus der Habsburgermonarchie in die USA behandelt, fünf der Schulbücher verweisen zudem auf die Binnenmigration innerhalb der Monarchie. Im Gegensatz zu diesen positiven Ergebnissen muss jedoch die Darstellung der Migrationsgeschichte des 20. und 21. Jahrhunderts sehr kritisch betrachtet werden, da sie sich als äußerst defizitär erweist. Beinahe alle analysierten Lehrwerke reduzieren die Migration im 20. und 21. Jahrhundert auf die transatlantische Emigration, die Gastarbeitermigrationen der 1960er- und 1970er-Jahre und die Fluchtbewegungen aus Syrien 2015. Dabei werden wesentliche Schlüsselereignisse der europäischen und österreichischen Migrationsgeschichte - insbesondere im Zusammenhang mit dem Zweiten Weltkrieg und Nationalsozialismus - nicht thematisiert, wodurch bei den Lernenden der Eindruck eines migrationslosen 20. Jahrhunderts entsteht. Die Situation der Displaced Persons wird in keinem einzigen Schulbuch angeführt und auch die Vertreibung der jüdischen Bevölkerung im Zuge des Nationalsozialismus findet sich nur in drei der Lehrwerke. Selbiges gilt für die Vertreibung der deutschsprachigen Minderheiten nach Ende des Zweiten Weltkrieges. Schlüsselereignisse in Zusammenhang mit dem Kalten Krieg - wie der Ungarnaufstand oder der Prager Frühling - werden in den Schulbüchern ebenso kaum thematisiert wie die EU-Binnenmigration im 21. Jahrhundert. Insgesamt erfüllt daher keines der analysierten Lehrwerke den Anspruch des Lehrplans, die „Migrationsgeschichte vom 19. Jahrhundert bis zur Gegenwart“ (BMBF, 2016, o.S.) zu behandeln.

\subsection{Migration als historisches und politisches Thema}

Im Lehrplan, der zwischen Modulen der (1) historischen, (2) historisch-politischen und (3) politischen Bildung unterscheidet, wird das Modul Migration rein der historischen Bildung zugeordnet. Dementsprechend findet sich in den analysierten Schulbüchern vor allem eine stark geschichtliche und weniger politische Auseinandersetzung mit dem Themenbereich. Doch auch die historische Perspektive auf Migration divergiert zwischen den einzelnen Lehrwerken stark, wie die bereits vorgestellten Ergebnisse der empirischen Erhebungen zeigen. Insbesondere variieren die dargestellten Migrationsereignisse. Durch die fehlende Zuordnung des Moduls zur politischen Bildung weisen die Lehrwerke nur ansatzweise Aspekte ebendieser auf, obwohl sich durch die im Lehrplan vorgegebene Behandlung von Migration im 21. Jahrhundert viele Aspekte der politischen Bildung ergeben.

Ausgehend von den geschichts- und politikdidaktischen Prinzipien (vgl. Hellmuth \& Kühberger 2016; Gautschi 2009; Pandel 2012; Sander 2014) kann festgestellt werden, dass die Wissenschaftsorientierung durch die Einbeziehung von wissenschaftlichen Definitionen und aktuellen Migrationszahlen gegeben ist. Hingegen können die Problemorientierung und Kontroversität als eher mangelhaft klassifiziert werden. Teilweise werden in den Schulbüchern zwar unterschiedliche Einstellungen und Positionen thematisiert, jedoch werden diese in weiterer Folge nicht durch Anregungen, die eine kontroverse Auseinandersetzung ermöglichen, aufgegriffen. Die Lebenswelt- und Subjektorientierung, die die Lebenswelt der Schüler*innen zum Ausgangspunkt des historischen und politischen Lernens macht, wird in allen Lehrwerken vertreten. Das subjektorientierte Lernen findet in vielen Schulbüchern in Kombination mit handlungsorientierten Aufgabenstellungen statt. Exemplarisch können hier die Aufforderungen, eine Migrationskarte der Klasse zu erstellen (vgl. L5, S. 126) oder mittels Stammbaum die eigene 
Migrationsbiographie zu beleuchten (vgl. L4, S. 90), genannt werden. Die Prozessorientierung ist hingegen in keinem der Lehrwerke zur Gänze gegeben. Zwar wird in den meisten Schulbüchern thematisiert, dass Migration ein Teil der Menschheitsgeschichte ist und seit jeher stattfindet, jedoch wird durch die lückenhafte Behandlung von Migration im 20. Jahrhundert der Eindruck erzeugt, als wäre Migration ein Phänomen des 19. Jahrhunderts und der Gegenwart, was aus Sicht einer prozessorientierten Geschichtsdidaktik unzufriedenstellend ist. Positiv ist an dieser Stelle zu erwähnen, dass fünf der sieben analysierten Lehrwerke trotz des fehlenden Lehrplanbezuges auch Migrationsprozesse vor dem 19. Jahrhundert thematisieren und somit zumindest teilweise zu einem Querschnitt der Migrationsgeschichte beitragen. Der Gegenwartsbezug der Lehrwerke ist durchaus gegeben, was sich aus der lehrplanmäßigen Festlegung der Behandlung von Migration im 21. Jahrhundert ergibt. Insbesondere die Fluchtbewegung des Jahres 2015 wird in allen untersuchten Schulbüchern behandelt. Aufgrund des historisch chronologischen Aufbaus der Lehrwerke wird Migration im 21. Jahrhundert in den meisten Lehrwerken jedoch erst am Ende des Kapitels bearbeitet, wodurch die im Gegenwartsbezug festgelegte Orientierung an gegenwärtigen Ereignissen als Ausgangspunkt für historisches Lernen in den meisten Lehrwerken nicht erfüllt wird. Das zentrale geschichts- und politikdidaktische Prinzip der Multiperspektivität, das vorsieht, dass der Unterricht den Lernenden ermöglicht, verschiedene Standpunkte und Sichtweisen einzunehmen und zu diskutieren, zeigt sich nur marginal repräsentiert, was insbesondere auf die nur ansatzweise Behandlung von Migration als politischem Thema zurückgeführt werden kann. Ansätze von Multiperspektivität lassen sich durch die Einbeziehung von schriftlichen Quellen, in denen Migrant*innen über ihr Schicksal berichten, feststellen, jedoch finden sich nur vereinzelt Aufgabenstellungen, die multiperspektivisch historisches und politisches Lernen ermöglichen.

Nach Betrachtung der geschichts- und politikdidaktischen Prinzipien kann subsumiert werden, dass die Wissenschaftsorientierung und der Lebensweltbezug in allen Lehrwerken in geeignetem Ausmaß vorhanden sind, während die Prozessorientierung und der Gegenwartsbezug nur partiell gegeben und die Kontroversität und Multiperspektivität nahezu nicht vorhanden sind. Es lässt sich zudem festhalten, dass trotz gewisser Ansätze der politischen Bildung die historische Auseinandersetzung mit dem Phänomen Migration überwiegt. In den Schulbüchern finden sich nur vereinzelt Textpassagen oder Aufforderungen, die ein politisches Lernen ermöglichen. Auch die Aufgabenstellungen werden vom historischen Lernen dominiert. Dementsprechend kann die Behandlung von Migration als politischem Thema in den Lehrwerken als nur ansatzweise vorhanden bezeichnet werden.

\section{Zusammenfassung: Erste Schritte eines langen Weges}

Insgesamt lassen sich zwischen den in diesem Beitrag analysierten, nach dem neuen, modularisierten Lehrplan 2016 verfassten Schulbüchern und jenen Ergebnissen, die aus der Studie von Hintermann et al. (2013) - welche den Ausgangspunkt für die Schulbuchanalyse bildete - hervorgingen, deutliche Veränderung feststellen. Diese manifestieren sich insbesondere in drei Bereichen: (1) der Thematisierung von Migration, (2) der Differenzierung verschiedener Migrationsformen und -ursachen sowie (3) der ansatzweisen Entproblematisierung von Migration.

Unter Thematisierung ist zu verstehen, dass im Zuge des modularisierten Lehrplans Migration erstmalig im GSPUnterricht der Sekundarstufe I systematisch behandelt wird, da eine entsprechende curriculare Verankerung vorliegt. Jedoch bleibt zu kritisieren, dass das entsprechende Modul im Lehrplan lediglich der historischen Bildung zugeordnet wurde, wenngleich bereits der Modultitel „Migration vom 19. Jahrhundert bis in die Gegenwart“ (BMBF, 2016) durch den Gegenwartsbezug sinnvollerweise auch eine politisch bildende Beschäftigung mit Migration ermöglichen müsste. Durch die Dominanz der historischen Bildung und die fehlende Thematisierung von zentralen politischen Debatten bezüglich Migration wird politisches Lernen im Themenbereich Migration in den analysierten Schulbüchern nur marginal ermöglicht, was jedoch stärker als Manko des Lehrplanes als jenes der Schulbücher zu betrachten ist. Zudem werden in den meisten Lehrwerken zentrale Migrationsereignisse des 20. Jahrhunderts - insbesondere, wenn sie in Zusammenhang mit dem Nationalsozialismus stehen - nicht thematisiert, wodurch der Eindruck eines migrationslosen 20. Jahrhunderts entsteht und das Geschichtsbild der Lernenden verfälscht wird. Daher werden die Lehrwerke dem Anspruch des Lehrplans, „Migration vom 19. Jahrhundert bis in die Gegenwart“ (BMBF, 2016) zu beleuchten, nicht gerecht. 
Der zweite große Unterschied betrifft die Differenzierung. Im Vergleich zu den Studienergebnissen von Hintermann et al. (2013), die eine undifferenzierte Darstellung von Migration in den Schulbüchern konstatierten, überzeugen alle analysierten Lehrwerke durch klare Begriffsdefinitionen von Migration. Im Großteil der Schulbücher wird zudem explizit oder implizit zwischen Migration und Flucht differenziert. In allen Lehrwerken wird eine Vielzahl an Migrationsursachen angeführt, wodurch Migration in ihrer Breite vermittelt wird. Einige der Lehrwerke führen zu diesem Zweck sogar Push- und Pull-Faktoren an, welche die Emigrations- bzw. Immigrationsmotive der Migrant*innen aufzeigen. Tiefergehende Ursachen der Migrationen, wie beispielsweise politische Instabilität im Herkunftsland oder demographische Entwicklungen im Aufnahmeland, werden hingegen nahezu nicht thematisiert. Auch wird die Binnenmigration - ebenso wie Migration als globales Phänomen - in einigen Lehrwerken nicht oder nur unzureichend behandelt.

Drittens kommt es schließlich in den meisten der analysierten Schulbücher zu einer schrittweisen Entproblematisierung von Migration, indem entweder ,Vorteile' und ,Herausforderungen' von Migration gegenübergestellt werden oder Migration als Chance thematisiert wird. Dies ist wiederum insofern problematisch, da Migrant*innen dadurch in einen Nützlichkeitsdiskurs (vgl. Geuenich, 2015; Ströhle, 2014) gedrängt werden und aufgrund ökonomischer oder demographischer Abwägungen in ,nützliche' und weniger ,nützliche' Migrant*innen unterteilt werden. Wesentlich seltener als die Darstellung von Migration als Chance oder Herausforderung findet sich in den Schulbüchern das Narrativ von Migration als etwas Selbstverständlichem, das als „menschliches Muster“ (Liebig, 2007, S. 7) den historischen Normal- und nicht den Sonderfall bildet.

Das deutlichste Ergebnis, das bereits im Zuge der Vorarbeiten zu den Schulbuchanalysen gewonnen werden konnte, stellt die Tatsache dar, dass Lehrwerke, welche nach dem Lehrplan 2008 verfasst wurden, Migration auf der 7. Schulstufe überhaupt nicht oder nicht systematisiert behandeln. Demgegenüber stehen die dem modularisierten Lehrplan 2016 (BMBF 2016) entsprechenden Schulbücher, welche Migration lehrplankonform als eigenes Modul bearbeiten. Alle der analysierten Lehrwerke, die nach dem Lehrplan 2016 verfasst wurden, gehen dabei auf unterschiedliche Weise auf die Themen ein, wobei der Aufbau der Werke einander stark ähnelt. Differenzen zwischen den für den Lehrplan 2016 lediglich adaptierten und den im Zuge der Lehrplanreform neu entstandenen Schulbüchern lassen sich zwar in Einzelfällen feststellen, jedoch erlauben sie keine allgemein gültige Schlussfolgerung über die Qualität der Behandlung von Migration in den adaptierten Schulbüchern im Vergleich zu den im Zuge der Lehrplanreform neu entstandenen Lehrwerken.

Trotz aller positiver Tendenzen im Vergleich zu den von 2011 bis 2013 analysierten Lehrwerken (Hintermann et al., 2013) zeigt die durchgeführte Schulbuchstudie einige Kritikpunkte auf. Die bereits kritisierte fehlende Zuordnung des Moduls zur politischen Bildung zeigt sich durch die nicht vorhandene Behandlung von Migration als politischem Thema in den Schulbüchern, wodurch Politiker*innen als Akteur*innen, die Migration begünstigen oder behindern können, sowie politische und gesellschaftliche Debatten über Migration kaum thematisiert werden. Einzelne Fallbeispiele zeugen zum Teil von deutlicher inhaltlicher Tiefe, die vermittelt wird, jedoch wird kein einziges der analysierten Schulbücher auch nur ansatzweise dem Anspruch gerecht, Migration „vom 19. Jahrhundert bis zur Gegenwart“ aufzuzeigen. Die abschließende Empfehlung lautet daher, Migration im Sinne eines Basiskonzepts (vgl. Germ, 2018) stärker als Querschnittsthematik von der 6. bis zur 8. Schulstufe zu verankern, um der Breite und Vielschichtigkeit der Thematik ohne Verzicht auf Fachwissen Rechnung tragen zu können und auch politisches Lernen zu ermöglichen. Dies würde jedoch eine entsprechende Adaptierung des Lehrplans voraussetzen, der bei all seinen aufgezeigten Schwächen immerhin der Migrationsthematik den Weg in die Schulbücher geebnet hat.

\section{Literatur}

BMBF (2015). Unterrichtsprinzip Politische Bildung. Grundsatzerlass 2015.

https://www.bmbwf.gv.at/Themen/schule/schulrecht/rs/1997-2017/2015 12.html

BMBF (2016). Lehrplan Geschichte und Sozialkunde/Politische Bildung. https://www.politiklernen.at/dl/mnoNJKJKonmomJgx4IJK/Gesetzesblatt 113. Verordnung 18 Mai 2016.pdf

Früh, W. (2017). Inhaltsanalyse. Theorie und Praxis (9. Aufl.). UVK Konstanz München. 
Gautschi, P. (2009). Guter Geschichtsunterricht. Grundlagen, Erkenntnisse, Hinweise. Wochenschau Schwalbach.

Germ, A. (2015). Konzeptuelles Lernen in der Politischen Bildung. Theoriebildung - Fachdidaktische Umsetzung - Praxisbeispiele. LIT Wien.

Geuenich, H. (2015). Migration und Migrant(inn)en im Schulbuch. Diskursanalysen nordrhein-westfälischer Politik- und Sozialkundebücher für die Sekundarstufe I. Springer Wiesbaden.

Hämmerle, K., Sandner, W. \& Sickinger, H. (2009): Politische Bildung in der Perspektive von Lehramtsstudierenden. In Österreichische Zeitschrift für Politikwissenschaft, 38 (Heft 3), S. 357-372.

Handro, S. \& Schönemann B. (Hrsg.) (2011). Geschichtsdidaktische Schulbuchforschung. LIT Berlin.

Hellmuth, T. \& Klepp, C. (Hrsg.) (2010). Politische Bildung. Geschichte-Modelle - Praxisbeispiele. Böhlau Wien Köln Weimar.

Hellmuth, T. \& Kühberger, C. (2016). Kommentar zum Lehrplan der Neuen Mittelschule und AHS-Unterstufe „Geschichte und Sozialkunde/Politische Bildung". https://www.politiklernen.at/dl/NqssJKJKonmomJqx4OJK/GSKPB Sek I 2016 Kommentar_zum Lehrplan_Stand $26 \quad 09201$ $\underline{6}$ pdf

Hintermann, C., Markom, C., Üllen, S. \& Weinhäupl, H. (2013). Debating Migration in Textbooks and Classrooms in Austria. In Jemms - Journal of Educational Media, Memory, and Society, S. 79-106.

Kipman, U. \& Kühberger, C. (2018). Einsatz und Nutzung des Geschichtsschulbuches. Eine Large-ScaleUntersuchung bei Schülern und Lehrern. Springer Wiesbaden.

Liebig, S. (2007). Migration und Weltgeschichte. Wochenschau Schwalbach.

Markom, C. \& Weinhäupl, H. (2013). Migration diskursiv: Problematisierung und Sprachkritik in Schulbuch und Schule. In ASSA-Austrian Studies in Social Anthropology, Sondernummer KSA-Tage 2013, S. 15-21.

Mayring, P. (2015a). Qualitative Inhaltsanalyse. Grundlagen und Techniken. Beltz Weinheim.

Mayring, P. (2015b). Qualitative Inhaltsanalyse. In Flick, U. et al. (Hrsg.): Qualitative Sozialforschung. Eine Einführung (11. Aufl.). Rowohlt Reinbeck (S. 468-475).

Pandel, H.-J. (2012). Geschichtsdidaktik. Wochenschau Schwalbach.

Sander, W. (2012). Wege zur Professionalisierung der Politischen Bildung - zwischen Fachlichkeit und Interdisziplinarität. In Diendorfer, G., Hellmuth, T. \& Hladschik, P.: Politische Bildung als Beruf. Professionalisierung in Österreich. Wochenschau Schwalbach (S. 49-62).

Sander, W. (Hrsg.) (2014). Handbuch Politische Bildung. Wochenschau Schwalbach.

SORA (Hrsg.) (2014). Politische BildnerInnen 2014. Politische Bildung in der Volksschule und Schulen der Sekundarstufe I in Wien. https://www.sora.at/fileadmin/downloads/projekte/2014 SORAEndbericht Politische BildnerInnen.pdf

Ströhle, P. (2014). Migration im Spiegel niedersächsischer Schulbücher der Fächer Erdkunde, Gemeinschaftskunde und Geschichte der gymnasialen Oberstufe. Masterarbeit Universität Osnabrück. http://library.fes.de/pdf-files/stufo/201505/stroehle 2014 masterarbeit pdf-a-version.pdf

Weinbrenner, P. (1995). Grundlagen und Methodenprobleme sozialwissenschaftlicher Schulbuchforschung. In Olechowski, R. (Hrsg.): Schulbuchforschung. Lang Wien Frankfurt (S. 21-45). 